Conserved behavioral and genetic mechanisms in the pre-hatching molt of the nematode Pristionchus pacificus

Lewis and Hong

( ) Biomed Central 


\title{
Conserved behavioral and genetic mechanisms in the pre-hatching molt of the nematode Pristionchus pacificus
}

Victor M Lewis and Ray L Hong ${ }^{*}$

\begin{abstract}
Background: During development, juvenile nematodes undergo four molts. Although the number of molts appears to be constant within the Nematoda, the timing of the first molt can occur either before or after hatching. A previous study indicates that, as in some parasitic nematode lineages, a pre-hatching juvenile stage also exists in Diplogastrid nematodes. A detailed description of these sequence of events has yet to be shown for any single species.

Findings: To delineate the timing of the pre-hatching molt in the beetle-associated Pristionchus pacificus, we tracked individual mid-J1 stage worms inside the eggshell through the J1-J2 transition and hatching. We found that active movement ended 21 hours after egg-laying, followed by lethargus and hatching. We inferred that lethargus behavior represents the onset of the first molt, which precedes each post-hatching molt in C. elegans and P. pacificus. The onset of the J1-J2 molt was also marked by the upregulation of the P. pacificus molting marker Ppa-pnhr-1. We further corroborated the pre-hatching molt with the isolation of two genetic mutants that exhibited aberrant molting both inside the egg and after hatching, as characterized by protracted and often-aborted shedding of the old cuticle.

Conclusion: Our results describe in detail the pre-hatching juvenile molt in P. pacificus, provide strong visual evidence of a pre-hatching molt, and show support for common genetic mechanisms regulating molting in the pre-hatching and post-hatching developmental stages. Our findings support the hypothesis that the evolution of pre-hatching development in Diplogastrid nematodes is likely due to a heterochronic shift between the timing of the first molt and hatching.
\end{abstract}

Keywords: Molting, Ecdysis, Developmental timing, Nematode, Heterochrony

\section{Findings}

Introduction

The Ecdysozoa is a clade of invertebrates, including nematodes and arthropods, which share the common process of shedding the exoskeleton or cuticle. The nematode cuticle is a multi-layered extracellular matrix that protects the entire body of the growing organism [1]. During transitions from one juvenile stage to another, the new cuticle emerges underneath and eventually replaces the old cuticle to accommodate the larger body of the next developmental stage. Molting starts

\footnotetext{
* Correspondence: ray.hong@csun.edu

Department of Biology, California State University, Northridge, 18111 Nordhoff Street, Northridge, CA 91330-8303, USA
}

with apolysis, or shedding of the old cuticle, followed by synthesis of the new cuticle, and culminates with ecdysis, or escape from the old cuticle. Nematodes undergo molting transition from active foraging behavior to lethargus, a quiescent period marked by a lack of movement during which the new cuticle is synthesized. The end of lethargus and the resumption of active behavior occur just prior to ecdysis, when the worm breaches the old cuticle [2].

Unlike arthropods, which can vary in both the timing and number of molts, all nematodes during larval development undergo four molts before reaching reproductive maturity [3]. This phylum-wide developmental constraint is likely not limited by genetic restrictions as single gene mutations in the model nematode Caenorhabditis elegans 
can cause changes to the molting cycle, such as supernumerary molts in lin-4, lin-14, and lin-29 mutants $[4,5]$. Although the four molts after hatching in C. elegans appear to be the ancestral phenotype, there are several examples that deviate from this pattern in plant and animal parasites [6]. Nematodes that molt prior to hatching include the parasitic pinewood nematode Bursaphelenchus xylophilus and the potato cyst nematode Globodera pallida $[3,7,8]$, as well as the vertebrate parasites Ascaris lumbricoides and Toxocara cati $[3,9]$. Among non-parasitic nematodes, larvae that undergo the first molt within the eggshell have been described in the Pristionchus and Oigolaimella species in the family Diplogastridae [10]. Because the evolution of parasitism in nematodes may involve stepwise changes between free-living, phoretic, necromenic, and parasitic lifestyles $[11,12]$, the $P$. pacificus necromenic lifestyle in close association with beetles invites studies on developmental plasticity, timing, and novelty. In particular, the presence of the pre-hatching molt within these diverse lineages hints at the role a prehatching larval stage may play in driving the evolution of parasitism.

$P$. pacificus was once thought to represent a unique group that possess only three juvenile stages prior to adulthood [6]. Subsequent investigation has since revealed that the first molt actually occurs prior to hatching in Diplogastrid nematodes, including P. pacificus [10]. Behavioral events that characterize this as a complete developmental stage have not been observed, but this observation is crucial for the understanding of developmental timing. In order to establish behavioral and genetic similarities between the pre-hatching and post-hatching molts in $P$. pacificus, we tracked development from late-stage embryos through the stage 1 juvenile (J1) to J2 transition and hatching. We further corroborated our observations by monitoring expression changes of a $P$. pacificus molting marker as well as obtaining general molting defective mutants that show both pre-hatching and post-hatching molting-defects. Our findings further support the hypothesis that the pre-hatching J1 stage in $P$. pacificus is homologous to the first post-hatching L1 stage in C. elegans.

\section{Results}

To delineate the sequence of events leading to the first molt in $P$. pacificus eggs, we tracked development from the middle of the first J1 juvenile stage until hatching in individual worms $(n=15)$. For convenience, we presumed the end of embryogenesis begins around 13 hours when the worm ceases to elongate and J1 cuticle synthesis is superficially complete $[10,13]$. Because molting is a dynamic process that is difficult to pinpoint directly using a single morphological feature, especially inside the confined space within the eggshell, we specifically looked for developmental lethargus that typically indicates the onset of molt in C. elegans. From 16 hours on, worms were observed for 10 minutes once every hour using Differential Interference Contrast (DIC) microscopy. Worms were considered active when movement occurred consistently throughout the 10-minute window, and in lethargus when there was minimal movement during the same time frame. Hatching was confirmed visually when the worm leaves its eggshell. We inferred that ecdysis occurred between the termination of lethargus and the onset of hatching, in rapid succession. Active behavior during the J1 stage of development ended $21.5 \pm 0.74$ hours post egg-laying, followed by lethargus $24.1 \pm 0.64$ hours post egg-laying, and hatching $25.1 \pm 0.64$ post egg-laying (Figure 1A; Additional file 1). Active behavior consisted of rapid movements, including full-body turns, interspaced by short periods of no movement. In contrast, worms in lethargus remained relatively motionless, with only slight repositioning movements during the approximately 2.5-hour lethargus period. Hatching was characterized by

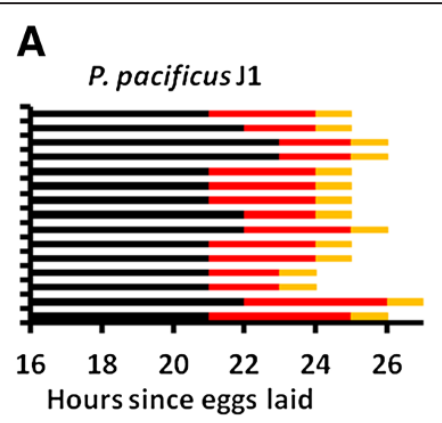

Active
B

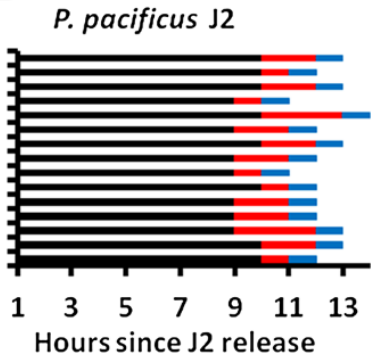

C C. elegans embryo

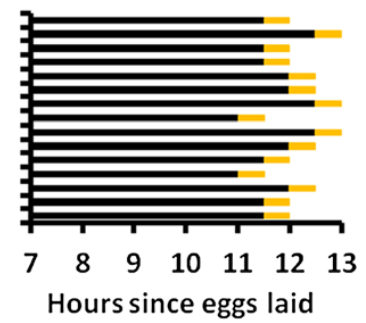

Lethargus

Hatching

Ecdysis

Figure 1 Pre-hatching juvenile (J1) development in P. pacificus. (A) Pre-hatching development in wild-type P. pacificus PS312. (B) Post-hatching development in $\mathbf{J} 2$ stage wild-type P. pacificus. The sequence of behaviors observed in (A) is almost identical to those in (B). (C) Late-embryonic development in wild-type C. elegans N2 lacks a lethargus period. 
rapid turning movements that culminated with ramming of the eggshell within one hour of the end of lethargus. Therefore, lethargus coincides with the timing of the prehatching molt similar to the molting behavior found in C. elegans.

To determine if the observed pre-hatching behaviors were similar to the behaviors associated with molting in post-hatching $P$. pacificus, we tracked an additional population of worms from the post-hatching J2 arrest through the J2-J3 molt. Active behavior ended $9.5 \pm 0.51$ hours after J2 release on food, followed by lethargus after $11.4 \pm$ 0.81 hours, and ecdysis after $12.4 \pm 0.81$ hours (Figure $1 \mathrm{~B}$ ). Active behavior consisted of typical feeding activity interspaced by short rests. Lethargus behavior was similarly characterized by a general lack of movement, although short movements, totaling no more than one body length, were occasionally observed (Additional file 2). Worms undergoing ecdysis were observed to resume activity with quick longitudinal movement coupled with whole-body turns, presumably to dislodge the previous cuticle. Thus, the post-hatching J2-J3 molt also exhibits the characteristic lethargus state found in the earlier J1-J2 molt inside the egg. To confirm that our observation is indeed restricted to Diplogastrid nematodes, we also tracked a population of $C$. elegans embryos from post-elongation embryogenesis through hatching. Active behavior during late-embryogenesis ended $11.8 \pm 0.50$ hours after egg laying and hatching occurred $12.3 \pm 0.52$ hours after egg laying (Figure 1C; Additional file 3). No lethargus period was observed. We also did not observe a possible molt inside the C. elegans eggshell that was reported to be limited to the pharyngeal cuticle [2]. In conclusion, we observed the same stereotyped behavioral events leading to a J1-J2 molt in the late-stage pre-hatching $P$. pacificus juveniles as those observed in newly hatched $P$. pacificus $\mathrm{J} 2$ juveniles and the molts in C. elegans.

To provide additional evidence that molting occurs inside the eggshell, we performed a genetic screen for general molting-defective mutants. We surmised that post-hatching molting-deficient mutants would also include eggs with deficiencies in the pre-hatching molt, given that many genetic regulators affect all molts [14]. We performed a non-saturating genetic screen for $P$. pacificus $\mathrm{F}_{2}$ juveniles with defective molting and isolated two complementing mutant strains, Ppa-mlt (csu26) and Ppa-mlt(csu28). The molting deficiency is easily identified by the intact shed cuticle surrounding both the anterior and posterior parts of the worm, whereas the cuticle of wild-type worms protrude only from the anterior end prior to ecdysis (Figure 2; Additional file 4). To assess the possibility of heterochronic changes in our mutant lines, we tracked Ppa-mlt(csu26) and Ppa-mlt(csu28)
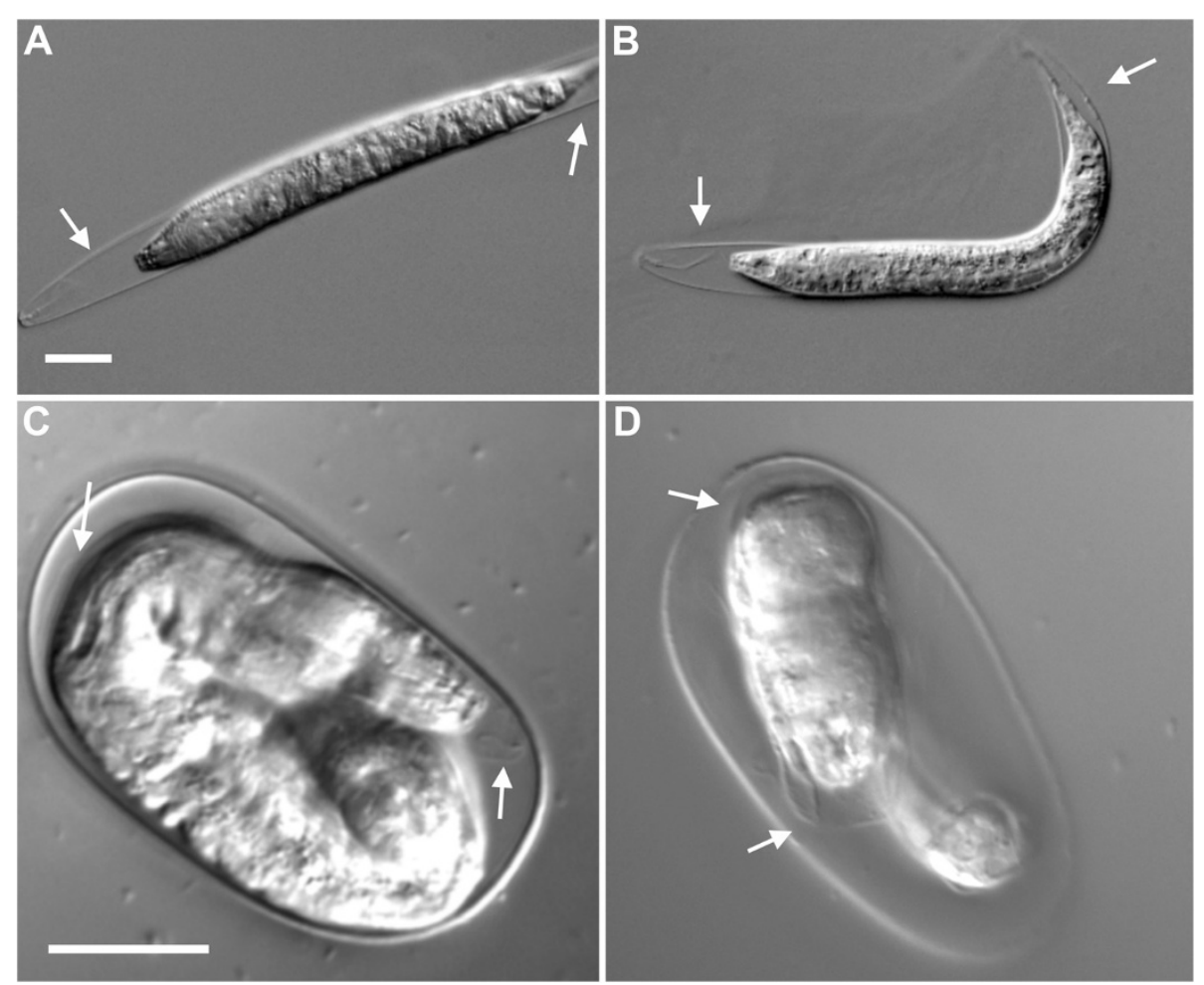

Figure 2 Molting-deficient phenotype of Ppa-m/t mutants. Post-hatching stage 2 juvenile (J2) to J3 molt (A) Ppa-mlt(csu26), and (B) Ppa-m/t (csu28). Pre-hatching molt (C) Ppa-mlt(csu26), and (D) Ppa-mlt(csu28). White arrows indicate unshed cuticle from previous stage. The unshed cuticle in the mutants can be observed in the posterior $(\mathbf{A}, \mathbf{B})$ and mid-body $(\mathbf{C}, \mathbf{D})$ regions - a phenotype not observed in wild-type. Scale bars $=20 \mu \mathrm{m}$. 
Table 1 Time to adulthood in Ppa-mlt mutants

\begin{tabular}{|c|c|c|c|c|c|c|c|c|c|}
\hline & $J 2$ & $\mathrm{J3}$ & $J 4$ & Adult & & $\mathrm{J} 2$ & $\mathrm{J3}$ & $J 4$ & Adult \\
\hline 0 hours & & & & & 48 hours & & & & \\
\hline Wild-type PS312 & 1.00 & - & - & - & Wild-type PS312 & - & - & 0.72 & 0.28 \\
\hline Ppa-m/t(csu26) & 1.00 & - & - & - & Ppa-mlt(csu26) & - & - & 0.88 & 0.12 \\
\hline Ppa-mlt(csu28) & 1.00 & - & - & - & Ppa-mlt(csu28) & - & 0.40 & 0.56 & 0.04 \\
\hline 12 hours & & & & & 60 hours & & & & \\
\hline Wild-type PS312 & 0.05 & 0.95 & - & - & Wild-type PS312 & - & - & - & 1.00 \\
\hline Ppa-mlt(csu26) & 0.13 & 0.87 & - & - & Ppa-mlt(csu26) & - & - & - & 1.00 \\
\hline Ppa-mlt(csu28) & 0.34 & 0.66 & - & - & Ppa-mlt(csu28) & - & 0.25 & 0.64 & 0.11 \\
\hline 24 hours & & & & & 72 hours & & & & \\
\hline Wild-type PS312 & - & 0.86 & 0.14 & - & Wild-type PS312 & - & - & - & 1.00 \\
\hline Ppa-mlt(csu26) & - & 0.98 & 0.02 & - & Ppa-mlt(csu26) & - & - & - & 1.00 \\
\hline Ppa-mlt(csu28) & - & 1.00 & - & - & Ppa-mlt(csu28) & - & - & 0.58 & 0.42 \\
\hline 36 hours & & & & & 84 hours & & & & \\
\hline Wild-type PS312 & - & 0.02 & 0.86 & 0.12 & Wild-type PS312 & - & - & - & 1.00 \\
\hline Ppa-mlt(csu26) & - & 0.09 & 0.87 & 0.04 & Ppa-mlt(csu26) & - & - & - & 1.00 \\
\hline Ppa-m/t(csu28) & - & 0.58 & 0.42 & - & Ppa-mlt(csu28) & - & - & - & 1.00 \\
\hline
\end{tabular}

Development from stage 2 juvenile (J2) arrest showing fraction per stage in PS312 ( $=55)$, csu26 ( $n=53)$, and csu28 ( $=45)$ after release from J2 arrest at 12-hour intervals. Individual mutants that arrested during a molt were not included in the total count.

from a synchronized $\mathrm{J} 2$ stage to adulthood and observed that although the time required to reach adulthood was longer in the csu28 line, all worms progressed through every stage and no molt was repeated or skipped (Table 1). The majority of post-hatching molting defects were observed in the J2-J3 molt, which is expected of mutations in genes responsible for a general molting process (Table 2). As no newly hatched $\mathrm{J} 2$ worms exhibited molting deficiencies, we hypothesize that Ppa-mlt juveniles that displayed the molting deficiency in the eggshell were unable to properly hatch. By subtracting the number of worms that hatched from those in the J1 stage, we inferred that the frequency of the pre-hatching molting deficiency is $11.43 \%$ and $16.38 \%$ for csu26 and csu28, respectively (Table 3 ). No wild-type worms showed pre-hatching molting defects using the same criteria.

Finally, to further substantiate our observations, we looked for gene expression patterns indicative of molting. Ppa-pnhr-1 is a previously identified homolog of the

Table 2 Frequency of molting deficient phenotype in Ppa-mlt mutants

\begin{tabular}{lcccc}
\hline & J2 & J3 & J4 & Total \\
\hline Wild-type PS312 & 0.004 & - & - & 0.004 \\
Ppa-mlt(csu26) & 0.201 & 0.011 & - & 0.213 \\
Ppa-mlt(csu28) & 0.092 & 0.020 & 0.006 & 0.118 \\
\hline
\end{tabular}

Stage-specific frequency of molting deficiency in PS312 $(n=1061)$, csu26 $(\mathrm{n}=800)$, and $\operatorname{csu} 28(\mathrm{n}=500)$. nuclear receptor ultraspiracle (Usp), a well characterized member of the arthropod molting pathway [15-17]. Ppa-pnhr-1 has been shown to have cyclical peaks of expression prior to all three post-hatching molts, and is the only known marker associated with the onset of molting in P. pacificus [18]. Using quantitative real-time PCR, we measured mRNA expression and found that Ppa-pnhr-1 level remained low in the early $\mathrm{J} 1$ followed by a distinct peak prior to the J1-J2 molt (Figure 3). As a negative control, we also assayed the Ppa-pnhr-1 expression profile at the J2 developmental arrest, a period which occurs immediately after hatching in the absence of food. During this arrested development, when we are certain that nematodes are not approaching the J2-J3 molt, the level of Ppa-pnhr-1 expression is much lower than 21-hour embryos and not significantly different from 16-hour embryos. The timing of Ppa-pnhr-1 peak expression coincides with our observation of lethargus shortly before the first molt inside the egg. The similarity of lethargus

Table 3 Pre-hatching molting-deficient phenotype in Ppa-m/t mutants

\begin{tabular}{lccc}
\hline & Viable J1 & Hatched & Inferred molt-deficient \\
\hline Wild-type PS312 & 0.93 & 0.93 & 0.00 \\
Ppa-mlt(csu26) & 0.71 & 0.60 & 0.11 \\
Ppa-mlt(csu28) & 0.50 & 0.33 & 0.17 \\
\hline
\end{tabular}

The pre-hatching frequency of the molting deficient phenotype was inferred in PS312 $(n=235), \operatorname{csu} 26(n=223)$, and $\operatorname{csu} 28(n=46)$. J1, stage 1 juvenile. 

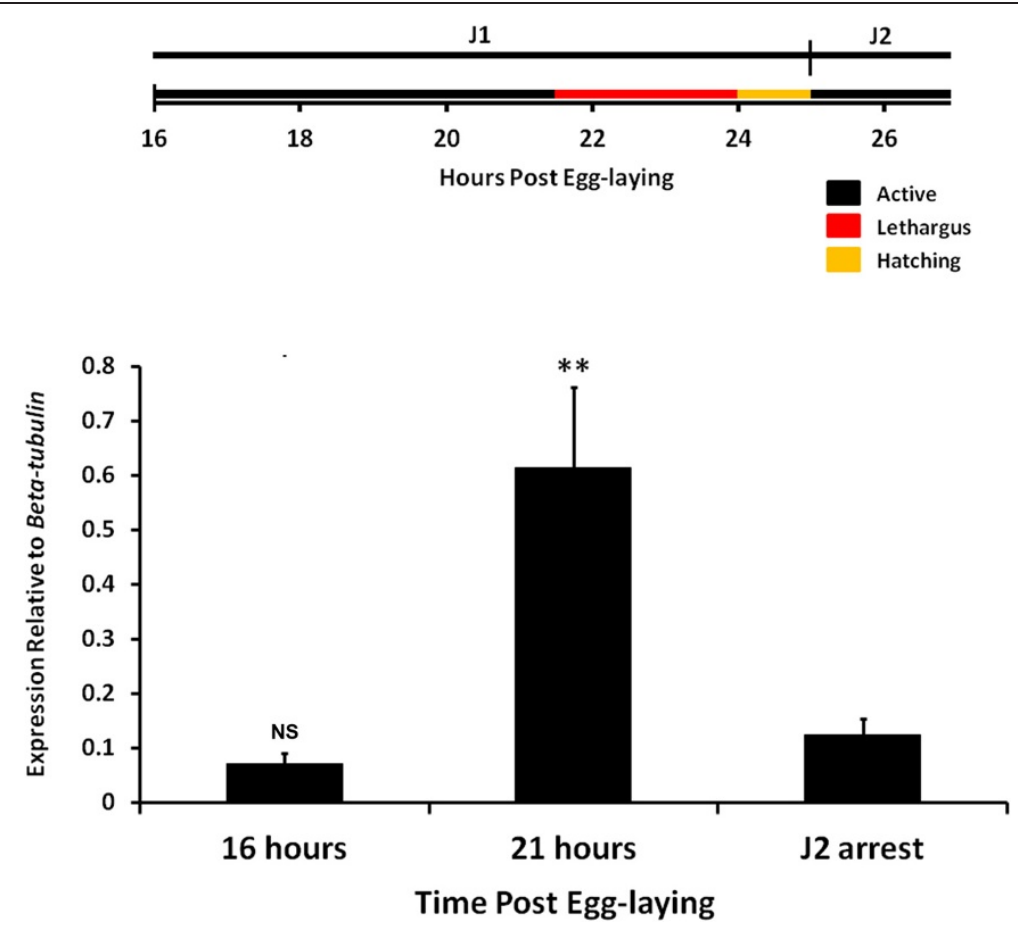

Figure 3 Expression of the nuclear receptor ultraspiracle (Usp) homolog Ppa-pnhr-1 is expressed prior to the first molt inside the egg. Expression is low in early stage 1 juvenile (J1) (16 hours) and high in late $\mathrm{J} 1$ (21 hours), indicative of a pending molt. The J2 developmental arrest serves as the negative control. Data represent eight total qPCR reactions from two independent CDNA samples at each time point. Each cDNA sample was collected from RNA from four pooled synchronized populations. The relative expression ratio of Ppa-pnhr-1 to Ppa-beta-tubulin was normalized to the no-reverse transcriptase negative control. Error bars represent standard error of the mean. ${ }^{* *} P \leq 0.01$ (Dunn's test to J2 arrest).

behavior and increased expression of the post-hatching molting marker Ppa-pnhr-1 strongly suggests that the molt inside the egg is a bonafide molting event.

\section{Conclusion}

We reveal for the first time the behavioral and gene expression events associated with the first molt inside the eggshell of $P$. pacificus. Our findings show the highly stereotypical lethargus behavior occurring in the prehatching molt as well as the J2 to J3 molt in P. pacificus, and support the hypothesis that the pre-hatching molt is homologous to the post-hatching molts in P. pacificus and C. elegans. The newly isolated Ppa-mlt mutants show molting defects both before and after hatching, indicating that the mutations are required for proper molting in all developmental stages. Upregulation of Ppa-pnhr-1 in pre-hatching J1 worms further corroborated our analysis. Interestingly, no homolog of Ppa-pnhr-1/Usp has been found in C. elegans and the functional role of Ppa-pnhr-1 has not been determined. We summarize our results with previously published data to offer a consensus model of pre-hatching development in P. pacificus [6,10] (Figure 4). Our study supports the hypothesis that the pre-hatching molt in P. pacificus is likely due to the result of a heterochronic shift in juvenile development and the timing of hatching [10].
The occurrence of the first molt prior to hatching in diverse plant and animal parasitic nematodes suggests multiple independent convergence events. One explanation may be that prolonging pre-hatching juvenile development before the infective dauer stage would provide selective advantage to parasitic nematodes. Alternatively, the co-occurrence of mouth dimorphism and prehatching molt in several genera within the Diplogastridae suggests that replacing the pharyngeal cuticle prior to hatching allows for the elaboration of the pharynx as a feeding organ during the course of evolution [10]. As J1 larvae are viable without the eggshell, identification of earlier hatching mutants may be a productive approach toward addressing the consequences of hatching as $\mathrm{J} 1$ larvae in P. pacificus.

Finally, we propose that the term, embryonic molt, be redefined as the pre-hatching molt in Diplogastrid nematodes, based on our observation that the pre-hatching molt occurs in the J1 to J2 transition just prior to hatching, when embryogenesis has already been completed. The results of our experiments have further characterized the pre-hatching development in P. pacificus with precise timing of lethargus as well as expression of Ppa-pnhr-1 to better define genes associated with the first molt. These findings promote future studies on the genetic mechanisms responsible for the identified 


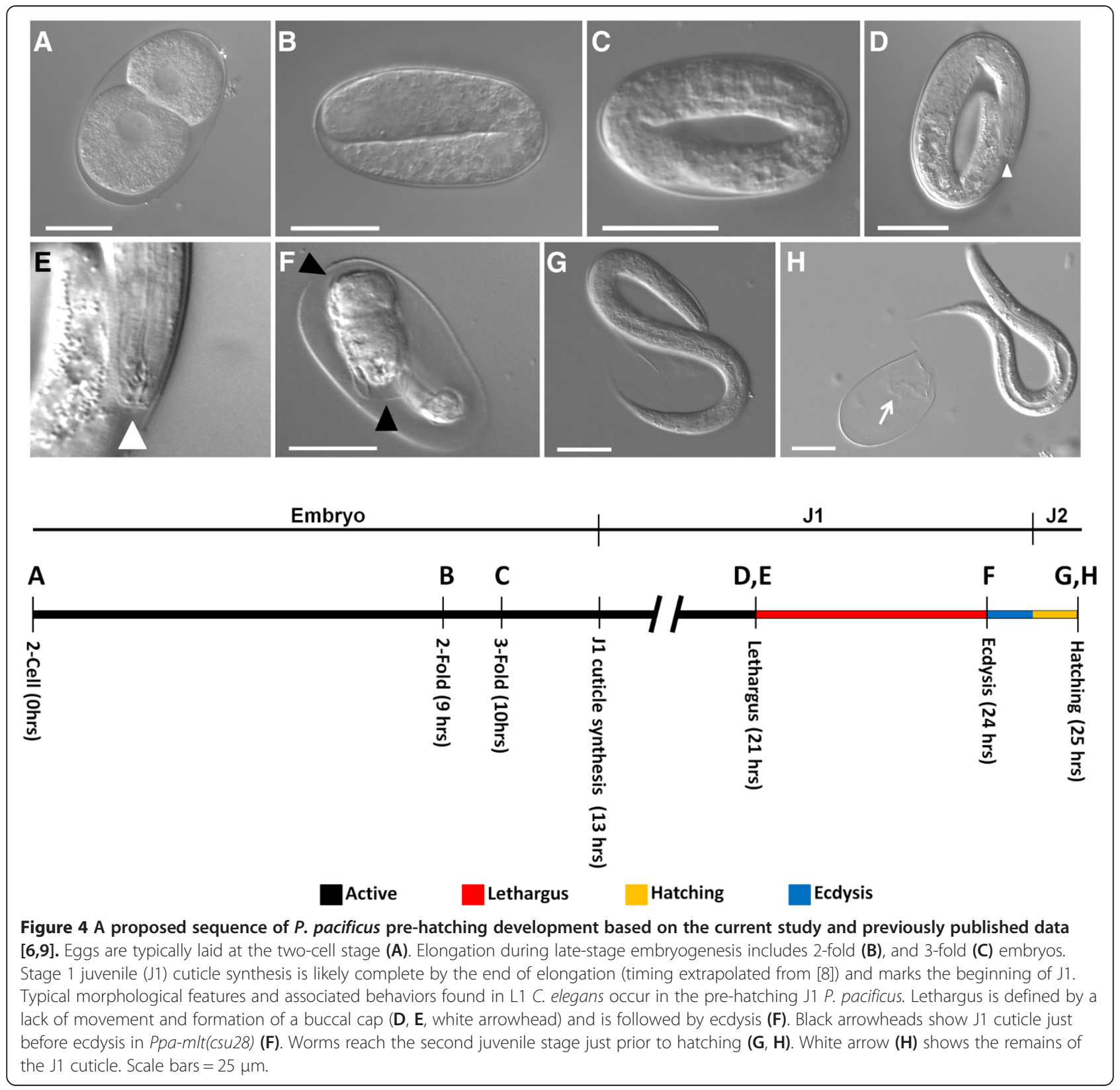

heterochronic changes in the timing of $P$. pacificus hatching, and may help to answer both how novelty occurs in developmental timing and how the presence of a pre-hatching developmental stage might contribute to the evolution of parasitism and the Diplogastrid dimorphic mouth formation.

\section{Materials and methods}

\section{Nematode and mutagenesis}

$P$. pacificus wild-type and emd mutant lines from the PS312 background were cultured at room temperature (approximately $23^{\circ} \mathrm{C}$ ) on Escherichia coli OP50 on nematode growth media [19]. Eggs were cultured at $20^{\circ} \mathrm{C}$ from synchronization until observation. Mutagenized strains were generated in the PS312 CA background using $50 \mathrm{mM}$ ENU; 1,500 F1 animals were cloned and their F2 progeny inspected for the presence of molting defects. From plates containing mutants, defective animals were picked again and screened once more for the mutant phenotype. Mutant lines were backcrossed three times to wild-type PS312 and homozygous molting-defect escapers were established for detailed analyses. 


\section{Quantitative real-time $P C R$ ( $q P C R$ )}

J2 larvae were synchronized at the J2 arrest by bleaching gravid hermaphrodites and allowing the remaining embryos to hatch on unseeded plates for 12 hours. Eggs were synchronized by allowing young adult hermaphrodites to lay eggs for 15 minutes on plates seeded with OP50 prior to removal. cDNA was synthesized from RNA in Trizol with Superscript III First-Strand Synthesis Kit (Invitrogen, Calrsbad, California). qPCR was performed and analyzed using SYBR chemistry on BioRad CFX96 (Hercules, California) with primers designed to span exon-intron boundaries (forward, reverse): Ppa-beta-tubulin, TCC AAGATCCGTGAGGAGTA, GGAGAGGGTGGCATTG TAG; Ppa-pnhr-1, CTCTTGAACGGCGTCCCTCTTC, GTGCAGAGTTGCGAAGGCTG. qPCR data represent four technical replicates from two independently isolated RNA samples at each time point. Each biological replicate was collected from four pooled synchronized populations. Relative expression ratio of Ppa-pnhr-1 to the reference gene Ppa-beta-tubulin was normalized to the no-reverse transcriptase negative control. Analysis of variance (ANOVA) was followed by the Dunn's test.

\section{Imaging}

Worms were observed using either a Leica S8E stereomicroscope or a Leica DM6000 (Solms, Germany) for DIC imaging and prepared using Adobe Photoshop (San Jose, California). Eggs were mounted on slides with egg buffer without anesthetics for observation of the J1 larvae. For post-hatching experimentation, worms were observed on NGM culture plates at $80 \times$ with a Leica S8E dissecting microscope. For imaging, hatched juveniles were paralyzed with $40 \mu \mathrm{M}$ sodium azide and mounted on slides with M9 buffer. Visual tracking of lethargus sequences in $P$. pacificus were made by observing synchronized embryos for 10 minutes every hour from 16 hours after egg laying until hatching. C. elegans embryos were tracked for 10 minutes every 30 minutes from 30 minutes after egg laying until hatching. Tracking of time until adulthood in Ppa-mlt alleles excludes those individuals that exhibited obvious molting defects. To visualize the presence of the pre-hatching molt in the mutants, worms from these lines were synchronized by egg-laying. When approximately $80 \%$ of larvae had hatched we screened the remaining eggs for pre-hatching juveniles that had not properly completed the molt.

\section{Statistical analysis and graphical representations}

Statistical analysis was performed using Excel (Microsoft, Redmond, Washington) and Instat software (GraphPad Software, San Diego, California). Graphical representations of collected data were prepared using Microsoft Excel, PowerPoint, and Adobe Photoshop.

\section{Additional files}

Additional file 1: $P$. pacificus active behavior. DIC video of stage 1 juvenile (J1) P. pacificus PS312 wildtype during active behavior. Time-lapse video compiled from images taken over a 1.5 hour timeframe approximately 6 hours prior to hatching.

Additional file 2: $\boldsymbol{P}$. pacificus lethargus behavior. DIC video of stage 1 juvenile (J1) P. pacificus PS312 wildtype during lethargus behavior. Time-lapse video compiled from images taken over a 1.5 hour timeframe approximately 3 hours prior to hatching.

Additional file 3: $\boldsymbol{C}$. elegans active behavior. DIC video of $C$. elegans N2 wildtype embryo during active behavior. Time-lapse video compiled from images taken over a 1-hour timeframe approximately 3 hours prior to hatching.

Additional file 4: Ppa-mlt(csu28) mutant molting phenotype. DIC Z-stack of the Ppa-m/t(csu28) molting deficiency in the stage 1 juvenile (J1) pre-hatching stage.

\section{Abbreviations}

J: juvenile stage; Usp: ultraspiracle.

\section{Competing interests}

The authors declare that they have no competing interests.

\section{Authors' contributions}

Experimental design: RLH and VML; performed experiments: VML; data analysis: RLH and VML; prepared manuscript: RLH and VML. Both authors read and approved the final manuscript.

\section{Acknowledgements}

We thank A Frand for helpful comments on this manuscript, R Mansfield and $\checkmark$ Guizar for assistance with qPCR, and C Gissendanner for the Ppa-pnhr-1 plasmid. Funding was provided by the Peter Bellinger Student Research Award (VML) and the CSUN Probationary Faculty Support Program (RLH).

Received: 3 July 2014 Accepted: 27 August 2014

Published: 19 September 2014

\section{References}

1. Aguinaldo AM, Turbeville JM, Linford LS, Rivera MC, Garey JR, Raff RA, Lake JA: Evidence for a clade of nematodes, arthropods and other moulting animals. Nature 1997, 387:489-493.

2. Singh R, Sulston J: Some observations on moulting in Caenorhabditis elegans. Nematologica 1978, 24:63-71.

3. Lee DL: The Biology of Nematodes. London: Taylor and Francis; 2002

4. Ambros $\mathrm{V}$ : A hierarchy of regulatory genes controls a larva-to-adult developmental switch in C. elegans. Cell 1989, 57:49-57.

5. Ambros V, Horvitz HR: Heterochronic mutants of the nematode Caenorhabditis elegans. Science 1984, 226:409-416.

6. Felix M, Hill R, Schwarz H, Sternberg PW, Sudhaus W, Sommer RS: Pristionchus pacificus, a nematode with only three juvenile stages, displays major heterochronic changes relative to Caenorhabditis elegans. Proc R Soc London 1999, 266:1617-1621.

7. Oh WS, Jeong PY, Joo HJ, Lee JE, Moon YS, Cheon HM, Kim JH, Lee YU, Shim YH, Paik YK: Identification and characterization of a dual-acting antinematodal agent against the pinewood nematode, Bursaphelenchus xylophilus. PLoS One 2009, 4:e7593.

8. Palomares-Rius JE, Jones JT, Cock PJ, Castillo P, Blok VC: Activation of hatching in diapaused and quiescent Globodera pallida. Parasitology 2013, 140:445-454.

9. Anderson RC: Nematode Parasites of Vertebrates. Their Development and Transmission. Wallingford: CAB International; 1992.

10. Fürst Von Lieven A: The embryonic moult in diplogastrids (Nematoda) homology of developmental stages and heterochrony as a prerequisite for morphological diversity. Zool Anzeiger - A J Comp Zool 2005, 244:79-91.

11. Dillman AR, Chaston JM, Adams BJ, Ciche TA: PLoS pathogens: an entomopathogenic nematode by any other name. PLoS Pathog 2012, 8:e1002527. 
12. Hong RL, Sommer RJ: Pristionchus pacificus: a well-rounded nematode. Bioessays 2006, 28:651-659.

13. Altun ZF, Herndon LA, Crocker C, Lints R, Hall DH: WormAtlas. [http://www.wormatlas.org]

14. Frand $A R$, Russel $S$, Ruvkun G: Functional genomic analysis of $C$. elegans molting. PLoS Biol 2005, 3:e312

15. Yao TP, Forman BM, Jiang Z, Cherbas L, Chen JD, McKeown M, Cherbas P, Evans RM: Functional ecdysone receptor is the product of EcR and Ultraspiracle genes. Nature 1993, 366:476-479.

16. Barchuk AR, Maleszka R, Simoes ZL: Apis mellifera ultraspiracle: CDNA sequence and rapid up-regulation by juvenile hormone. Insect Mol Biol 2004, 13:459-467.

17. Hannan GN, Hill RJ: Lcusp, an ultraspiracle gene from the sheep blowfly, Lucilia cuprina: cDNA cloning, developmental expression of RNA and confirmation of function. Insect Biochem Mol Biol 2001, 31:771-781.

18. Parihar M, Minton RL, Flowers S, Holloway A, Morehead BE, Paille J, Gissendanner CR: The genome of the nematode Pristionchus pacificus encodes putative homologs of RXR/Usp and EcR. Gen Comp Endocrinol 2010, 167:11-17.

19. Brenner S: The genetics of Caenorhabditis elegans. Genetics 1974, 77:71-94.

doi:10.1186/2041-9139-5-31

Cite this article as: Lewis and Hong: Conserved behavioral and genetic mechanisms in the pre-hatching molt of the nematode Pristionchus pacificus. EvoDevo 2014 5:31.

\section{Submit your next manuscript to BioMed Central and take full advantage of:}

- Convenient online submission

- Thorough peer review

- No space constraints or color figure charges

- Immediate publication on acceptance

- Inclusion in PubMed, CAS, Scopus and Google Scholar

- Research which is freely available for redistribution 